Chirurg 2015 $\cdot 86: 990$

DOI 10.1007/s00104-015-0075-z

Online publiziert: 11. August 2015

c) Springer-Verlag Berlin Heidelberg 2015

CrossMark

\section{Haeder · J. Jähne}

Klinik für Allgemein- und Viszeralchirurgie, Schwerpunkt für endokrine und onkologische

Chirurgie, Diakoniekrankenhaus Henriettenstiftung gGmbH, Hannover, Deutschland

\title{
Einfluss passagerer Metallstents bei stenosierendem Ösophaguskarzinom auf das onkologische Outcome
}

\section{Originalpublikation}

Marierre C, Gronnier C, Duhamel A et al (2015) Self-expanding covered metallic stent as a bridge to surgery in esophageal cancer: impact on oncologic outcomes. J Am Coll Surg 220:287-296

\section{Hintergrund}

Dysphagie und Malnutrition sind häufige Symptome bei Diagnose eines (meist schon fortgeschrittenen) Ösophaguskarzinoms (EC) mit negativen Auswirkungen auf die multimodale Therapie, Morbidität und Mortalität sowie Prognose. Es existieren verschiedene Optionen zur Verbesserung des Ernährungsstatus mit allerdings auch assoziierten Komplikationen respektive reduzierter Lebensqualität. Die Implantation selbstexpandierender beschichteter Metallstents (SEMS) ist ein mittlerweile etabliertes und weitgehend sicheres Verfahren insbesondere in der Palliativsituation und ermöglicht eine zügige Wiederherstellung der oralen Nahrungsaufnahme, die Langzeiteffekte in der kurativen Situation sind bisher aber nicht bekannt. Ziel dieser Multicenterstudie war die Evaluation des Einflusses der „neoadjuvanten“S Stentimplantation auf das onkologische Outcome.

\section{Methodik}

Die Daten von insgesamt 2279 Patienten, die im Zeitraum von 2000 bis 2010 in 30 französischsprachigen Zentren eine kurativ intendierte operative Therapie bei EC (inklusive Adenokarzinome des ösopha- gogastralen Überganges Typ I und II nach Siewert) erhielten, wurden retrospektiv nach spezifischen Kriterien (u. a. Dysphagie und Malnutrition) analysiert und selektiert. Alle Patienten mit SEMS-Implantation $(n=38)$ wurden mittels PropensityScore-Analyse 1:4 einer Kontrollgruppe ohne SEMS-Implantation ( $n=152)$ zugeordnet. Die prätherapeutische Diagnostik sowie das therapeutische Vorgehen orientierten sich an den gültigen Leitlinien. Das mediane Follow-up betrug 43,6 Monate und war in beiden Gruppen vergleichbar.

\section{Ergebnisse}

Im Vergleich der SEMS- mit der Kontrollgruppe betrug die Morbidität 63,2 vs. $59,2 \%(p=0,658)$ sowie die Mortalität 13,2 vs. $8,5 \%(p=0,370)$, allerdings waren die schweren postoperativen Komplikationen in der SEMS-Gruppe höher (44,7 vs. $27,0 \%, p=0,033)$. Die R0-Resektionsrate war nach Stent-Implantation signifikant geringer ( 71,0 vs. $85,5 \%, p=0,041)$, zudem lagen hier trotz in beiden Gruppen initial vergleichbarer Tumorstadien lokal fortgeschrittenere Tumoren vor. Es zeigte sich eine kürzere rezidivfreie Zeit in der SEMS-Gruppe (median 6,5 Monate vs. 9,0 Monate, $p=0,049$ ) sowie ein geringeres medianes "overall survival" von 17,4 vs. 23,1 Monaten $(p=0,023)$. Damit korrelierte auch die reduzierte 3-JahresÜberlebensrate von 28 vs. $40 \%(p=0,043)$.

\section{Diskussion und Fazit}

Trotz der bekannten Einschränkungen einer retrospektiven Untersuchung mit einem gewissen Selektions-Bias sowie auch der geringen Fallzahl in der SEMSGruppe schlussfolgern die Autoren aus den vorliegenden Daten, dass die SEMSImplantation in einer kurativ-intendierten Therapiesituation beim EC einen signifikant negativen Einfluss auf die R0Resektionsrate und das onkologische Outcome zu haben scheint. Ähnliche Ergebnisse konnten bereits in einer früheren Untersuchung zur überbrückenden SEMS-Implantation beim stenosierenden linksseitigen Kolonkarzinom nachgewiesen werden. Obgleich zur adäquaten Evaluation die Durchführung einer randomisierten Multicenterstudie erforderlich wäre, sollte hinsichtlich der hier genannten Effekte die "neoadjuvante“ Stentimplantation dennoch kritisch beurteilt und auch nur unter Berücksichtig alternativer Optionen (perkutane endoskopische Gastrostomie, Katheterjejunostomie) im Rahmen multidisziplinärer Therapieplanungen erwogen werden.

\section{Korrespondenzadresse}

\section{Haeder}

Klinik für Allgemein- und Viszeralchirurgie, Schwerpunkt für endokrine und onkologische Chirurgie

Diakoniekrankenhaus Henriettenstiftung gGmbH

Marienstr. 72-90, 30171 Hannover

Lars.Haeder@ddh-gruppe.de

Interessenkonflikt. L. Haeder und J. Jähne geben an, dass kein Interessenkonflikt besteht. 